Felder, G. and A. P. Veselov. (2007) “Polynomial Solutions of the Knizhnik-Zamolodchikov Equations and Schur-Weyl Duality,"

International Mathematics Research Notices, Vol. 2007, Article ID rnm046, 21 pages.

doi:10.1093/imrn/rnm046

\title{
Polynomial Solutions of the Knizhnik-Zamolodchikov Equations and Schur-Weyl Duality
}

\author{
Giovanni Felder ${ }^{1}$ and Alexander P. Veselov ${ }^{2}$ \\ ${ }^{1}$ Department of Mathematics, ETH Zurich, 8092 Zurich, Switzerland and \\ ${ }^{2}$ Department of Mathematical Sciences, Loughborough University, \\ Loughborough LE11 3TU, UK and Landau Institute for Theoretical \\ Physics, Moscow, Russia
}

Correspondence to be sent to: Giovanni Felder, Department of Mathematics, ETH Zurich, 8092 Zurich, Switzerland.e-mail: giovanni.felder@math.ethz.ch

An integral formula for the solutions of Knizhnik-Zamolodchikov (KZ) equation with values in an arbitrary irreducible representation of the symmetric group $S_{N}$ is presented for integer values of the parameter. The corresponding integrals can be computed effectively as certain iterated residues determined by a given Young diagram and give polynomials with integer coefficients. The derivation is based on Schur-Weyl duality and the results of Matsuo on the original $S U(n) \mathrm{KZ}$ equation. The duality between the spaces of solutions with parameters $m$ and $-m$ is discussed in relation with the intersection pairing in the corresponding homology groups.

\section{Introduction}

Let $G$ be a finite Coxeter group, $R$ be the corresponding root system, $m_{\alpha}, \alpha \in R$ be a system of multiplicities, which is a $G$-invariant function on $R$. Let $W$ be an irreducible representation of $G$ and define the Knizhnik-Zamolodchikov equation (KZ equation)

Received November 3, 2006; Revised April 16, 2007; Accepted May 22, 2007

Communicated by Igor Krichever

See http://www.oxfordjournals.org/our_journals/imrn/for proper citation instructions.

(C) The Author 2007. Published by Oxford University Press. All rights reserved. For permissions, please e-mail:journals.permissions@oxfordjournals.org. 
related to $W$ as the following system

$$
\partial_{\xi} \psi=\sum_{\alpha \in R_{+}} m_{\alpha} \frac{(\alpha, \xi)}{(\alpha, z)}\left(s_{\alpha}+1\right) \psi,
$$

where $s_{\alpha}$ are the corresponding reflections acting on $W$-valued functions $\psi(z)$. If the multiplicities $m_{\alpha}$ are integers, then all the solutions of the corresponding systems are homogeneous polynomials (see $[11,3]$ ) of degree equal to the value of the central element $\sum_{\alpha \in R_{+}} m_{\alpha}\left(s_{\alpha}+1\right)$ in the irreducible representation $W$. The finding of these solutions is an important part of the description of the so-called $m$-harmonic polynomials [2]. In the paper [3] these solutions were found explicitly in the simplest case of the standard (reflection) representation of $G=S_{N}$.

The main result of the present paper is an explicit integral formula for the solutions of the corresponding KZ equation

$$
\partial_{i} \psi=m \sum_{j \neq i}^{N} \frac{s_{i j}+1}{z_{i}-z_{j}} \psi, \quad i=1, \ldots, N
$$

with values in an arbitrary irreducible representation of the symmetric group $S_{N}$ for any positive integer $m$. Our approach is based on Schur-Weyl duality and the results of Matsuo, who found some integral formulas for the solutions of the original $S U(n) \mathrm{KZ}$ equation [10] inspired by the work of Fateev and Zamolodchikov [13] and Christe and Flume [1].

The main construction is the following. Let $\lambda$ be the Young diagram with $N$ boxes and $n$ rows of lengths $\lambda_{1}, \ldots, \lambda_{n}$ with $\lambda_{1} \geq \cdots \geq \lambda_{n}>0$. It is well known that for any such diagram one can construct an irreducible representation $W^{\lambda}$ of the symmetric group $S_{N}$ and any irreducible representation of $S_{N}$ can be described in this way (see e.g. [6]).

The space $W^{\lambda}$ has a basis $V_{T}$ labeled by the set $\mathcal{T}(\lambda)$ of all standard tableaux on $\lambda$, which are the numberings $T: \lambda \rightarrow\{1, \ldots, N\}$ of the boxes of $\lambda$, increasing from left to right and from top to bottom.

A fundamental set of solutions of the $\mathrm{KZ}$ equation with values in $W^{\lambda}$ can be also labeled by the set $\mathcal{T}(\lambda)$ :

$$
\psi_{T}\left(z_{1}, \ldots, z_{N}\right)=\sum_{T^{\prime} \in \mathcal{T}(\lambda)} \psi_{T, T^{\prime}}\left(z_{1}, \ldots, z_{N}\right) V_{T^{\prime}}
$$


The components $\psi_{T, T^{\prime}}\left(z_{1}, \ldots, z_{N}\right)$ are known to be polynomial in $z_{1}, \ldots, z_{N}$ [3]. We can give now an explicit formula for $\psi_{T, T^{\prime}}\left(z_{1}, \ldots, z_{N}\right)$ as an integral

$$
\psi_{T, T^{\prime}}=\int_{\sigma_{T}} \omega_{T^{\prime}}
$$

of some rational differential form $\omega_{T^{\prime}}$ over a certain cycle $\sigma_{T}$ in the top homology of the following configuration space $C_{\lambda}\left(z_{1}, \ldots, z_{N}\right)$ related to Young diagram $\lambda$.

For given $\lambda=\left(\lambda_{1}, \ldots, \lambda_{n}\right)$ let us define the integers $m_{i}, i=1, \ldots, n$ from the relation $\lambda=\left(m_{0}-m_{1}, m_{1}-m_{2}, \ldots, m_{n-2}-m_{n-1}, m_{n-1}\right)$. Explicitly we have

$$
m_{0}=\lambda_{1}+\lambda_{2}+\cdots+\lambda_{n}=N, m_{1}=\lambda_{2}+\cdots+\lambda_{n}, \ldots, m_{n-2}=\lambda_{n-1}+\lambda_{n}, m_{n-1}=\lambda_{n},
$$

so that $m_{s}$ is the number of boxes in the rows of $\lambda$ strictly lower than $s$.

Consider $n$ finite sets $X_{0}, X_{1}, \ldots, X_{n-1}$ of points on the complex plane $\mathbb{C}$ consisting of $m_{0}, \ldots, m_{n-1}$ points respectively with the condition that $X_{i}$ and $X_{i+1}$ have no common points for all $i=1, \ldots, n-2$. Let us denote the elements of $X_{0}$ as $z_{1}, \ldots, z_{N}$ and fix them. The corresponding configuration space of all admissible $\left\{X_{1}, \ldots, X_{n-1}\right\}$ is our $C_{\lambda}\left(z_{1}, \ldots, z_{N}\right)$. It has the dimension

$$
d_{\lambda}=\sum_{i=1}^{n-1} m_{i}=\sum_{r=1}^{n}(r-1) \lambda_{r}
$$

and can be described as the following subset in $\mathbb{C}^{d_{\lambda}}$ :

$$
C_{\lambda}\left(z_{1}, \ldots, z_{N}\right)=\left\{t_{s}^{b} \in \mathbb{C}, b \in \lambda, 1 \leq s \leq r(b)-1: t_{s+1}^{b} \neq t_{s}^{b^{\prime}}, t_{1}^{b} \neq z_{k}\right\},
$$

where we have denoted the elements of $X_{s}$ as

$$
X_{s}=\left\{t_{s}^{b} \in \mathbb{C}, b \in \lambda, r(b)>s\right\},
$$

and $r(b)$ is the row which the corresponding box $b$ belongs to.

On this space $C_{\lambda}\left(z_{1}, \ldots, z_{N}\right)$ we have a natural action of the group $G_{\lambda}=S_{m_{1}} \times$ $S_{m_{2}} \times \cdots \times S_{m_{n-1}}$. The cycles $\sigma_{T}$ in the top homology group $H_{t o p}\left(C_{\lambda}\left(z_{1}, \ldots, z_{N}\right)\right)$ can be defined for any numbering $T$ of $\lambda$, namely any bijection $T: \lambda \rightarrow\{1, \ldots, N\}$. Consider first the product $\Gamma_{T}$ of circles consecutively surrounding anti-clockwise $z_{k}$ with the variables $t_{1}^{b}, \ldots, t_{r(b)-1}^{b}, b=T^{-1}(k)$ located on these circles:

$$
\Gamma_{T}=\left\{t_{s}^{b} \in \mathbb{C}:\left|t_{s}^{b}-z_{k}\right|=\epsilon S, b=T^{-1}(k)\right\}
$$


for any real positive $\epsilon$ small enough. The corresponding cycle $\sigma_{T}$ is the skew-symmetrization of $\Gamma_{T}$ by the action of $G_{\lambda}$ :

$$
\sigma_{T}=\sum_{g \in G_{\lambda}}(-1)^{g} g_{*}\left(\Gamma_{T}\right)
$$

where $(-1)^{g}$ denote the sign of $g$, which is the product of signs of the corresponding permutations in $S_{m_{i}}$.

The form $\omega_{T}$ has the form

$$
\omega_{T}=\frac{1}{(2 \pi i)^{d_{\lambda}}} \Phi_{\lambda}^{m} \phi_{T} d t
$$

where

$$
\begin{aligned}
& \Phi_{\lambda}=\prod_{i<j}^{N}\left(z_{i}-z_{j}\right)^{2} \prod_{s, b \neq b^{\prime}}\left(t_{s}^{b}-t_{s}^{b^{\prime}}\right)^{2} \prod_{s, b, b^{\prime}}\left(t_{s+1}^{b}-t_{s}^{b^{\prime}}\right)^{-1} \prod_{k, b}\left(t_{1}^{b}-z_{k}\right)^{-1}, \\
& \phi_{T}=\prod_{s, b}\left(t_{s+1}^{b}-t_{s}^{b}\right)^{-1} \prod_{b}\left(t_{1}^{b}-z_{T(b)}\right)^{-1}
\end{aligned}
$$

and $d t=\prod_{s, b} d t_{s}^{b}$ is the exterior product of the differentials of all the coordinates (the order is not essential since it is only changes sign).

Theorem 1.1. For any given positive integer $m$, the integral formulas (1.2), (1.3) with the cycles $\sigma_{T}$ and forms $\omega_{T}, T \in \mathcal{T}(\lambda)$ defined above give a basis in the space of solutions of the KZ equation (1.1) with values in the irreducible $S_{N}$-module $W^{\lambda}$. The integral (1.3) can be effectively computed as an iterated residue and gives a polynomial in $z_{1}, \ldots, z_{N}$ with integer coefficients.

We have derived these formulas from the results of Matsuo [10] using the SchurWeyl duality. The fact that in such a way we get all the solutions of the corresponding $\mathrm{KZ}$ equation does not follow from [10] and needs to be proven independently. Using representation theory it turns out that it is sufficient to prove that one of the integrals $\psi_{T, T^{\prime}}$ does not vanish identically. This we prove by evaluating $\psi_{T, T}$ for the tableau assigning $k$ to the $k$ th box, counted from left to right and from top to bottom, in the asymptotic region $0 \ll\left|z_{1}\right| \ll \cdots \ll\left|z_{N}\right|$. We find

$$
\psi_{T, T}(z) \sim C \prod_{b \in \lambda} z_{T(b)}^{m(T(b)-1+c(b)-r(b))}+\cdots,
$$


for some integer $C \neq 0$, see Lemma 3.7, where $c(b), r(b)$ are the coordinates (column and row number) of the box $b \in \lambda$. As a by-product, we obtain a new derivation of the interesting formula, due to Frobenius [5] (see [9], exercise 7 in Chapter I and comment on p. 134) for the value $f_{2}(\lambda)$ of the central element $\sum_{i<j} s_{i j}$ in the representation $W^{\lambda}$ :

$$
f_{2}(\lambda)=\sum_{b \in \lambda}(c(b)-r(b))
$$

Theorem 1.1 applies to the case of positive integer $m$. The case of negative $m$ can be reduced to it by using the isomorphism between the space of solutions $K Z(V, m)$ of the $\mathrm{KZ}$ equation with values in the representation $V$ and parameter $m$ and the space $K Z(V \otimes$ Alt,$-m)$, where Alt $=\mathbb{C} \epsilon$ is the alternating representation. Indeed, it is easy to check that if $\psi \in K Z(V, m)$ then $\phi=\prod_{i>j}\left(z_{i}-z_{j}\right)^{-2 m} \psi \otimes \epsilon \in K Z(V \otimes$ Alt, $-m)$. In particular it follows that for negative $m$ all solutions are rational functions. In the last section we discuss also the duality between $K Z(V, m)$ and $K Z\left(V^{*},-m\right)$ given by the canonical map

$$
K Z(V, m) \otimes K Z\left(V^{*},-m\right) \rightarrow \mathbf{C}
$$

in relation with the intersection pairing in the corresponding homology groups.

The case we consider can be viewed as a very degenerate limit of the general theory of hypergeometric solutions of KZ equations associated with Kac-Moody algebras, see [12] and references therein. We should mention that similar integral formulas were found also by Kazarnovski-Krol [8] in the theory of zonal spherical functions of type $A_{n}$, but combinatorics of the corresponding configuration space is very different and not related to Young diagrams.

\section{Schur-Weyl duality}

We start with the classical Schur-Weyl duality between the representations of the general linear and symmetric groups.

Let $V$ be an $n$-dimensional complex vector space. Then the symmetric group $S_{N}$ on $N$ letters acts on the $N$-fold tensor product $V^{\otimes N}=V \otimes \cdots \otimes V$ by permutations of factors and this action commutes with the diagonal action of $G L(V)$. The Schur-Weyl theorem states that, as a $G L(V) \times S_{N}$ module, $V^{\otimes N}$ has a decomposition into a direct sum

$$
V^{\otimes N} \cong \oplus_{\lambda} M^{\lambda} \otimes W^{\lambda}
$$


where $M^{\lambda}$ are inequivalent irreducible $G L(V)$-modules and $W^{\lambda}$ are inequivalent irreducible $S_{N}$-modules. The sum is over partitions of $N$ into at most $n$ parts, namely sequences of integers $\lambda_{1} \geq \cdots \geq \lambda_{n} \geq 0$ with $\sum \lambda_{i}=N$.

Moreover if $n \geq N$ all irreducible $S_{N}$ modules appear. Thus we can realize every irreducible $S_{N}$-module as

$$
W^{\lambda}=\operatorname{Hom}_{G L(V)}\left(M^{\lambda}, V^{\otimes N}\right),
$$

for any $V$ of dimension $\geq N$.

An explicit description of $W^{\lambda}$ is obtained from the description of $M^{\lambda}$ as a highest weight module and depends on a choice of basis of $V$. Namely, let us fix a basis $e_{1}, \ldots, e_{n}$ of $V$ and introduce the decomposition $\mathfrak{g l}(V)=\mathfrak{n}_{-} \oplus \mathfrak{h} \oplus \mathfrak{n}_{+}$of the Lie algebra of $G L(V)$ into strictly lower triangular, diagonal and strictly upper triangular $n \times n$ matrices. For any $\mathfrak{g l}(V)$-module $E$ and any $\mu \in \mathfrak{h}^{*}=\mathbb{C}^{n}$, denote by $E_{\mu}=\{v \in E \mid X \cdot V=\mu(x) V, \forall x \in \mathfrak{h}\}$ the weight subspace of weight $\mu$. The space of primitive vectors of weight $\mu$ in $E$ is

$$
E_{\mu}^{\mathfrak{n}_{+}}=\left\{v \in M_{\mu} \mid a \cdot v=0, \forall a \in \mathfrak{n}_{+}\right\} .
$$

The irreducible module $M^{\lambda}$ is uniquely characterized by having a nonzero primitive vector $v_{\lambda}$ of weight $\lambda$, which is unique up to normalization. Moreover $M^{\lambda}$ is generated over $U\left(\mathfrak{n}_{-}\right)$by $v_{\lambda}$ and thus

$$
M^{\lambda}=\mathbb{C V}_{\lambda} \oplus \mathfrak{n}_{-} M^{\lambda} .
$$

An element of $\operatorname{Hom}_{G L(V)}\left(M^{\lambda}, V^{\otimes N}\right)$ is then uniquely determined by the image of the primitive vector and we obtain an isomorphism of $S_{N}$-modules

$$
W^{\lambda}=\left(V^{\otimes N}\right)_{\lambda}^{\mathfrak{n}_{+}}
$$

From this realization we obtain a basis of $W^{\lambda}$ labeled by standard Young tableaux, making connection to the classical construction of $W^{\lambda}$ as a Specht module, see [6]. Here is how it works: let $\lambda$ also denote the Young diagram with $N$ boxes with rows of lengths $\lambda_{1}, \ldots, \lambda_{m}$. To each numbering $T: \lambda \rightarrow\{1, \ldots, N\}$ of the boxes we associate a weight vector $e_{T}=e_{\alpha_{1}} \otimes \cdots \otimes e_{\alpha_{N}} \in\left(V^{\otimes N}\right)_{\lambda}$ so that $\alpha_{k}=i$ whenever $T^{-1}(k)$ is in the $i$ th row. For example, if $T$ is the numbering 


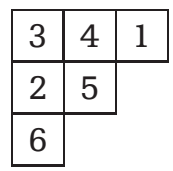

of $\lambda=(3,2,1)$, then $e_{T}=e_{1} \otimes e_{2} \otimes e_{1} \otimes e_{1} \otimes e_{2} \otimes e_{3}$.

The symmetric group $S_{N}$ acts on the set of numberings of $\lambda$ and we have $\sigma e_{T}=e_{\sigma T}$ for any $\sigma \in S_{N}$. For any numbering $T$ of $\lambda$, the row group $R(T)$ is the subgroup of $S_{N}$ consisting of permutations preserving the image of each row. Similarly, we have the column group $C(T) \subset S_{N}$. Two numberings $T, T^{\prime}$ give the same weight vector if and only $T^{\prime}=\sigma T$ for some $\sigma \in R(T)$. In this case $R(T)=R\left(T^{\prime}\right)$ and we say that $T$ and $T^{\prime}$ are row equivalent. Thus the vectors $e_{T}$ are associated to row equivalence classes $\{T\}$ of numberings of $\lambda$, which are called tabloids on $\lambda$. Recall that a standard tableau on $\lambda$ is a numbering of $\lambda$ which is increasing from left to right and from top to bottom.

Proposition 2.1. Let $\lambda$ be a partition of $N$ and $\operatorname{let} \operatorname{dim}(V) \geq N$.

1. The vectors $e_{T}$, where $\{T\}$ runs over tabloids on $\lambda$, form a basis of the weight space $\left(V^{\otimes N}\right)_{\lambda}$.

2. The vectors $V_{T}=\sum_{\sigma \in C(T)} \operatorname{sign}(\sigma) e_{\sigma T}$, where $T$ runs over the set $\mathcal{T}(\lambda)$ of standard tableaux on $\lambda$, form a basis of the $S_{N}$-module $W^{\lambda}=\left(V^{\otimes N}\right)_{\lambda}^{\mathrm{n}_{+}}$ of primitive vectors of weight $\lambda$.

For proofs see, e.g., Chapter 9 of [7].

A dual realization of $W^{\lambda}$ is also relevant. The symmetric bilinear form on $V$ for which the $e_{i}$ form an orthonormal basis induces a symmetric nondegenerate bilinear form $\langle$,$\rangle on each weight space \left(V^{\otimes N}\right)_{\lambda}$. If $\lambda$ is a partition of $N$, the tensors $e_{T}$, where $\{T\}$ runs over the set of tabloids on $\lambda$, are then an orthonormal basis of $\left(V^{\otimes N}\right)_{\lambda}$. Let $\tau$ be the antiautomorphism of $\mathfrak{g l}(V)$ given by matrix transposition with respect to the basis $e_{i}$. Then $\langle x \cdot V, w\rangle=\langle V, \tau(x) \cdot w\rangle, x \in \mathfrak{g l}(V)$. Moreover the bilinear form is $S_{N}$-invariant: $\langle\sigma \cdot V, \sigma \cdot w\rangle=\langle V, w\rangle, \sigma \in S_{N}, V, w \in V^{\otimes N}$.

Proposition 2.2. Let $\lambda$ be a partition of $N$ and let $\operatorname{dim}(V) \geq N$.

(i) The form $\langle$,$\rangle induces a nondegenerate S_{N}$-invariant pairing

$\left(V^{\otimes N} / \mathfrak{n}_{-} V^{\otimes N}\right)_{\lambda} \otimes\left(V^{\otimes N}\right)_{\lambda}^{\mathfrak{n}_{+}} \rightarrow \mathbb{C}$.

Thus we can identify $\left(V^{\otimes N} / \mathfrak{n}_{-} V^{\otimes N}\right)_{\lambda}$ with the dual $S_{N}$-module $\left(W^{\lambda}\right)^{*}$. 
(ii) The basis dual to the basis $V_{T}$ of $W^{\lambda}$ is given by the classes of the vectors $e_{T}$,

$$
T \in \mathcal{T}(\lambda) \text { in }\left(W^{\lambda}\right)^{*}=\left(V^{\otimes N} / \mathfrak{n}_{-} V^{\otimes N}\right)_{\lambda} .
$$

Proof. It follows from (2.2) and the complete reducibility of $V^{\otimes N}$ into a direct sum of irreducible highest weight modules that

$$
\left(V^{\otimes N}\right)_{\lambda}=\left(V^{\otimes N}\right)_{\lambda}^{\mathfrak{n}_{+}} \oplus\left(\mathfrak{n}_{-} V^{\otimes N}\right)_{\lambda} .
$$

Moreover this is an orthogonal direct sum with respect to the contravariant form, since $\tau$ maps $\mathfrak{n}_{-}$to $\mathfrak{n}_{+}$. Therefore the pairing is well-defined and is nondegenerate. Since $V_{T}(T \in \mathcal{T}(\lambda))$ form a basis of $W^{\lambda}$ and $e_{T}$ occurs in $V_{T}$ with coefficient 1 , we get $\left\langle e_{T}\right.$ $\left.\bmod \mathfrak{n}_{-}, v_{S}\right\rangle=\delta_{T, S}, T, S \in \mathcal{T}(\lambda)$. Thus the classes of $e_{T}$ form the dual basis of the dual module.

Remark 2.3. Actually, $S_{N}$-modules are self-dual, $\left(W^{\lambda}\right)^{*} \cong W^{\lambda}$ but the expression of the isomorphism with respect to the bases labeled by tableaux is nontrivial. The space of cycles in our integral formulae are more naturally associated with $\left(W^{\lambda}\right)^{*}$.

\section{Integral representation of solutions}

3.1 The action of the symmetric group on the solution space

We fix a Young diagram $\lambda$ and a positive integer $m$ and keep the notations of the introduction.

The KZ operators $D_{i}=\partial_{i}-m \sum_{j \neq i}\left(s_{i j}+1\right) /\left(z_{i}-z_{j}\right)$ appearing in (1.1) are commuting first-order holomorphic differential operators acting on $W^{\lambda}$-valued functions on the configuration space $C_{N}=\mathbb{C}^{N}-\cup_{i<j}\left\{z_{i}=z_{j}\right\}$. Thus the space of holomorphic solutions on any connected open subset $U \subset C_{N}$ has dimension $\operatorname{dim} W^{\lambda}$. The symmetric group $S_{N}$ acts on $C_{N}$ and thus on the functions with values in the $S_{N}$-module $W^{\lambda}$ by $(g \cdot \psi)(z)=$ $g\left(\psi\left(g^{-1} \cdot z\right)\right)$. The KZ operators obey $g \cdot D_{i} \psi=D_{g(i)} g \cdot \psi$ for all $g \in S_{N}$. Therefore $g \in S_{N}$ maps solutions on $U$ to solutions on $g \cdot U$. In particular we have an action of $S_{N}$ on the space of global solutions

$$
K Z(\lambda, m)=\left\{\text { holomorphic functions } \psi: C_{N} \rightarrow W^{\lambda}: D_{i} \psi=0, i=1, \ldots, N\right\}
$$

In fact, all local solutions extend to global solutions: 
Theorem 3.1. [11, 3] All local solutions of the Knizhnik-Zamolodchikov equation (1.1) with $m \in \mathbb{Z}_{>0}$ extend to homogeneous polynomials of degree $m\left(f_{2}(\lambda)+(N-1) N / 2\right)$, where $f_{2}(\lambda)$ is the value of the central element $\sum_{i<j} s_{i j}$ of the group algebra of $S_{N}$ in the representation $W^{\lambda}$. Moreover, the space of solutions $K Z(\lambda, m)$ is isomorphic to $W^{\lambda}$ as an $S_{N}$-module.

The homogeneity follows directly from the equations: if $\psi$ is a solution then $\sum z_{i} D_{i} \psi=\left(\sum z_{i} \partial_{i}-m\left(f_{2}(\lambda)+(N-1) N / 2\right)\right) \psi=0$.

\subsection{Matsuo's integral formulae.}

The configuration spaces $C_{\lambda}(z), z \in C_{N}$, of (1.4) form a fiber bundle over $C_{N}$ and the action of $S_{N}$ on the base lifts canonically to an action on the bundle. Indeed, $C_{\lambda}\left(z_{1}, \ldots, z_{N}\right)$ does not depend on the ordering of the $z_{i}$. The forms $\omega_{T}$ of eqn. (1.7) are holomorphic differential forms on the total space that restrict to holomorphic top differential forms $\omega_{T}(z)$ on each fiber $C_{\lambda}(z)$. They are defined for any numberings $T$, not just for standard tableaux and, by construction, they obey $\omega_{g T}(g \cdot z)=\omega_{T}(z)$ for all $g \in S_{N}$, where $g T=g \circ T$ is the natural action on the set of numberings $T: \lambda \rightarrow\{1, \ldots, N\}$.

Let $H_{\text {top }}\left(C_{\lambda}(z)\right)_{s}, z=\left(z_{1}, \ldots, z_{N}\right) \in C_{N}$, be the skew-symmetric part of the homology of the top degree $d_{\lambda}$ under the action of $G_{\lambda}=S_{m_{1}} \times \cdots \times S_{m_{n-1}}$ :

$$
H_{\text {top }}\left(C_{\lambda}(z)\right)_{s}=\left\{\sigma \in H_{\text {top }}\left(C_{\lambda}(z)\right): g_{*} \sigma=(-1)^{g} \sigma, \quad g \in G_{\lambda}\right\}
$$

Lemma 3.2. If $\sigma \in H_{\text {top }}\left(C_{\lambda}(z)\right)_{s}$ then $\int_{\sigma} \omega_{T}(z)$ depends only on the tabloid $\{T\}$ of $T$.

Indeed, if $T$ and $T^{\prime}$ differ by an element $h$ of the row group $R(T)$, inducing a permutation $h_{1}$ of the set of boxes of $\lambda$ then $\omega_{T}(z)=(-1)^{g} g^{*} \omega_{T^{\prime}}(z)$, where $g \in G^{\lambda}$ is the permutation $t_{i}^{b} \mapsto t_{i}^{h_{1}(b)}$ of the variables (the sign comes from the volume form $d t$ ).

The following result can be extracted from Matsuo's paper [10].

Theorem 3.3. (Matsuo [10]) Let $\sigma \in H_{t o p}\left(C_{\lambda}(z)\right)_{s}, \psi_{\sigma}(z)=\sum_{\{T\}} \int_{\sigma} \omega_{T}(z) e_{T} \in\left(V^{\otimes N}\right)_{\lambda}$, with summation over all tabloids $\{T\}$ on $\lambda$.

(i) $\psi_{\sigma}(z) \in W^{\lambda}=\left(V^{\otimes N}\right)_{\lambda}^{\mathfrak{n}_{+}}$.

(ii) As $z$ varies in some neighborhood of a point in $C_{N}, \psi_{\sigma}(z)$ is a solution of the KZ equation (1.1).

Recall that homology groups of neighboring fibers of a fiber bundle are canonically identified, so (ii) makes sense. 
Here are some details on how to deduce Theorem 3.3 from [10]: Matsuo considers the general Knizhnik-Zamolodchikov equation

$$
\partial_{i} \varphi=m \sum_{j \neq i} \frac{\Omega_{i, j}}{z_{i}-z_{j}} \varphi
$$

for $\varphi\left(z_{1}, \ldots, z_{N}\right)$ taking values in a tensor product $V_{\lambda_{1}}^{*} \otimes \cdots \otimes V_{\lambda_{N}}^{*}$ of Verma modules $V_{\lambda_{i}}^{*}$ over $\mathfrak{s l}_{n}$ of highest weight $\lambda_{i}{ }^{1}$. In this version of the $\mathrm{KZ}$ equation, $\Omega_{i j}$ denotes the action on the $i$-th and $j$-th factors of the tensor in $\mathfrak{s l}_{n} \otimes \mathfrak{s l}_{n}$ dual to the invariant bilinear form $(x, y)=\operatorname{tr}(x y)$ on $\mathfrak{s l}_{n}$. The Verma module $V_{\lambda}^{*}$ is generated by a highest weight vector $V_{\lambda}^{*}$ and is free over the Lie subalgebra of strictly lower triangular matrices $\mathfrak{n}_{-}$, so it has a Poincaré-Birkhoff-Witt basis

$$
u_{\lambda}^{*}(\vec{p})=\prod_{\alpha>\beta} \frac{E_{\alpha, \beta}^{p_{\alpha, \beta}}}{p_{\alpha, \beta} !} v_{\lambda}^{*}, \quad \vec{p}=\left(p_{\alpha, \beta}\right)_{n \geq \alpha>\beta \geq 1}
$$

where the product is defined for some choice of ordering of the standard basis $\left(E_{\alpha, \beta}\right)_{\alpha>\beta}$, of $\mathfrak{n}_{-}$. Accordingly, we have a basis $u^{*}(\vec{p})=\otimes_{a=1}^{N} u_{\lambda_{a}}^{*}\left(\vec{p}_{a}\right), \vec{p}=\left(\vec{p}_{1}, \ldots, \vec{p}_{N}\right)$, of the tensor product. Matsuo's integral formula for solutions has the form $\varphi=\sum I(\vec{p}) u^{*}(\vec{p})$ for some integrals $I(\vec{p})$ whose integrand is described explicitly in [10]. The sum is over all $\vec{p}$ such that $u^{*}(\vec{p})$ has a given weight $\lambda$. It is then shown in [10] that the solution takes values in the primitive vectors of weight $\lambda$.

For our purpose we need a special case of Matsuo's construction, for which the formulae simplify considerably. The vector representation $V$ of $\mathfrak{s l}_{n}$ is the quotient of the Verma module $V_{\varpi_{1}}^{*}$ with fundamental highest weight $\varpi_{1}$ by its maximal proper submodule. Thus we take $\lambda_{1}=\cdots=\lambda_{N}=\varpi_{1}$, and we have the tensor product $\pi_{N}:\left(V_{\varpi_{1}}^{*}\right)^{\otimes N} \rightarrow V^{\otimes N}$ of canonical projections. The KZ equation (3.1) makes sense for the tensor product of any $N$ modules; moreover any solution taking values in $\left(V_{\varpi_{1}}^{*}\right)^{\otimes N}$ projects to a solution taking values in $V^{\otimes N}$. In the latter case we have $\Omega_{i j}=S_{i j}-\frac{1}{n}$ Id, where $S_{i j}$ exchanges the $i$-th and the $j$-th factor of the tensor product and thus coincides with the action of $s_{i j}$ on the $S_{N}$-module $V^{\otimes N}$. It follows that if $\varphi$ is a solution of (3.1), then

$$
\psi\left(z_{1}, \ldots, z_{N}\right)=\prod_{i<j}\left(z_{i}-z_{j}\right)^{m(1+1 / n)} \pi_{N} \circ \varphi\left(z_{1}, \ldots, z_{N}\right)
$$

\footnotetext{
${ }^{1}$ Actually, he uses lowest weights instead of highest weights, $N-1$ instead of $N$ and $n+1$ instead of $n$, but it is easy to translate to our convention.
} 
is a solution of (1.1). The canonical projection $\pi: V_{\varpi_{1}}^{*} \rightarrow V$ sends most PBW basis vectors to zero. The only remaining ones are the classes of

$$
e_{\alpha}=E_{\alpha, \alpha-1} \ldots E_{3,2} E_{2,1} V_{\varpi_{1}}, \quad \alpha=1, \ldots, n .
$$

It is straightforward to check that we obtain our integral formulae, as described in the Introduction, by restricting the sum in Matsuo's formula to include only tensor products of vectors $e_{\alpha}$.

\section{Corollary 3.4.}

(i) The solution $\psi_{\sigma}$ of Theorem 3.3 can be written as

$$
\begin{gathered}
\psi_{\sigma}(z)=\sum_{T \in T(\lambda)} \int_{\sigma} \omega_{T}(z) V_{T} . \\
\text { (ii) } \psi_{\sigma}(g \cdot z)=g \psi_{\sigma}(z), \sigma \in H_{t o p}\left(C_{\lambda}(z)\right)_{s}=H_{t o p}\left(C_{\lambda}(g \cdot z)\right)_{s} .
\end{gathered}
$$

Proof. (i) follows from Theorem 3.3, (i) and Proposition 2.1. For (ii) one uses the original expression for $\psi_{\sigma}$.

\subsection{Completeness}

We show here that all solutions are given as integrals over suitable cycles. The proof is in two parts: first we construct an $S_{N^{-}}$equivariant map $\left(W^{\lambda}\right)^{*} \rightarrow K Z(\lambda, m)$, defined as the integral over the cycles $\sigma_{T}$. Since $\left(W^{\lambda}\right)^{*}$ is irreducible and of the right dimension, it then suffices to check that the map is nonzero, which can be done by an asymptotic analysis.

We start by describing the action of the symmetric group on cycles.

Lemma 3.5. For any numbering $T$ of $\lambda$ and $z=\left(z_{1}, \ldots, z_{N}\right) \in C_{N}$, let $\sigma_{T}=\sigma_{T}(z) \in$ $H_{t o p}\left(C_{\lambda}(z)\right)_{s}$ be the homology class defined in the introduction as the skew-symmetrization of the image of the fundamental class by a map $\left(S^{1}\right)^{d_{\lambda}} \rightarrow C_{\lambda}(z)$.

(i) For all $g \in S_{N}, \sigma_{g T}(g \cdot z)=\sigma_{T}(z)$.

(ii) If $T$ and $T^{\prime}$ are numberings of $\lambda$ differing by a row permutation, then $\sigma_{T}(z)=$ $\sigma_{T^{\prime}}(z)$. Thus $\sigma_{T}(z)$ depends only on the tabloid of $T$.

Proof. (i) holds by construction. The proof of (ii) is the same as the proof of Lemma 3.2. This time the sign comes from the change of orientation. 
Thus we get a map

$$
\begin{aligned}
\Psi_{m}^{\lambda}:\left(V^{\otimes N}\right)_{\lambda} & \rightarrow K Z(\lambda, m) \\
e_{T} & \mapsto \int_{\sigma_{T}} \omega=\sum_{T^{\prime} \in T(\lambda)} \psi_{T, T^{\prime}} V_{T^{\prime}}
\end{aligned}
$$

It is well defined since $\sigma_{T}$, just as $e_{T}$, depends only on the tabloid of $T$.

Lemma 3.6. The map $\Psi_{m}^{\lambda}$ is $S_{N}$-equivariant.

This follows from Lemma 3.5 and Corollary 3.4.

To prove that the map is nonzero we will use the following key technical lemma. Let $(r(b), c(b))$ be the coordinates (row and column number) of the box $b$ in the Young diagram $\lambda$.

Lemma 3.7. Let $T$ be the standard tableau mapping the $k$ th box, counted from left to right and top to bottom, to $k$. Then $\psi_{T, T}(z)$ is not identically zero. The leading term for $0 \ll\left|z_{1}\right| \ll\left|z_{2}\right| \ll \cdots \ll\left|z_{N}\right|$ is

$$
\psi_{T, T}(z) \sim C \prod_{b \in \lambda} z_{T(b)}^{m(T(b)-1+c(b)-r(b))}+\cdots
$$

for some $C \neq 0$.

Proof. We show that, as $z_{N} \rightarrow \infty$,

$$
\psi_{T, T}\left(z_{1}, \ldots, z_{N}\right)=C^{\prime} z_{N}^{m\left(N-1+\lambda_{n}-n\right)}\left(\psi_{T^{\prime}, T^{\prime}}\left(z_{1}, \ldots, z_{N-1}\right)+O\left(z_{N}\right)\right),
$$

where $T^{\prime}$ is the standard tableau with $N-1$ boxes obtained from $T$ by removing the last box and $C^{\prime}$ is a nonzero combinatorial factor. Since $\psi_{[1,1}=1$ for the tableau with one box, this gives an inductive proof of the claim.

Let $\lambda^{\prime}$, the shape of $T^{\prime}$, be $\lambda$ without the last box. Then

$$
\begin{aligned}
& \Phi_{\lambda}=\Phi_{\lambda^{\prime}} \prod_{k=1}^{N-1}\left(z_{k}-z_{N}\right)^{2}\left(t \frac{\sqrt{k}}{1}-z_{N}\right)^{-1} \prod_{s=1}^{n-1}\left(t_{s}^{\underline{N}}-t \frac{N]}{s-1}\right)^{-1} \\
& \prod_{s=1}^{n-1}\left(\prod_{\substack{k<N \\
r(\underline{k})>s}}\left(t_{s}^{\underline{k}}-t_{s}^{[N]}\right)^{2} \prod_{\substack{k<N \\
r(\underline{k})>s+1}}\left(t_{s+1}^{[k]}-t_{s}^{[N]}\right)^{-1} \prod_{\substack{k<N \\
r(\underline{k})>s-1}}\left(t_{s}^{[N]}-t_{s-1}^{\underline{k})^{-1}}\right),\right.
\end{aligned}
$$


where $k=T^{-1}(k)$ is the box of $\lambda$ labeled by $k$ and we set $t_{0}^{k}=z_{k}$. Also,

$$
\phi_{T}=\phi_{T^{\prime}} \prod_{s=1}^{n-1}\left(t_{s}^{N}-t_{s-1}^{N}\right)^{-1} .
$$

The leading term as $z_{N} \rightarrow \infty$ in $\psi_{T, T}$ is obtained when the variables $t_{S}^{N}$ run over circles around $z_{N}$. With the variable substitution $t_{s}^{\mathbb{N}}=z_{N}+\tau_{1}+\cdots+\tau_{s}$, the leading term as $z \rightarrow \infty$ of the integration of $\omega_{T}$ over the variables $t \frac{N}{S}$ is

$$
\pm \omega_{T^{\prime}} z_{N}^{m(N-1)} \operatorname{res}_{\tau_{n-1}=0} \cdots \operatorname{res}_{\tau_{1}=0} \Omega
$$

where

$$
\begin{aligned}
\Omega=\prod_{s=1}^{n-2}\left(z_{N}+\tau_{1}+\cdots+\tau_{s}\right)^{m\left(2 m_{s}-m_{s-1}-m_{s+1}\right)} \tau_{s}^{-m-1} d \tau_{s} \\
\quad \times\left(z_{N}+\tau_{1}+\cdots+\tau_{n-1}\right)^{m\left(2 m_{n-1}-m_{n-2}-1\right)} \tau_{n-1}^{-m-1} d \tau_{n-1} \\
=\prod_{s=1}^{n-2}\left(z_{N}+\tau_{1}+\cdots+\tau_{s}\right)^{m\left(\lambda_{s+1}-\lambda_{s}\right)} \tau_{s}^{-m-1} d \tau_{s} \\
\quad \times\left(z_{N}+\tau_{1}+\cdots+\tau_{n-1}\right)^{m\left(\lambda_{n}-\lambda_{n-1}-1\right)} \tau_{n-1}^{-m-1} d \tau_{n-1} .
\end{aligned}
$$

The residues can be computed explicitly. Such expressions give a nonzero result if the total power of all factors containing any given $\tau_{s}$ is negative. This power is $\left(\lambda_{n}-\lambda_{s}-1\right) m$ which is indeed negative for all $s=1, \ldots, n-1$.

Theorem 3.8. The map $\Psi_{m}^{\lambda}:\left(V^{\otimes N}\right)_{\lambda} \rightarrow K Z(\lambda, m)$ is an epimorphism of $S_{N}$-modules with $\operatorname{kernel}\left(\mathfrak{n}_{-} V^{\otimes N}\right)_{\lambda}$ and therefore defines an isomorphism $\left(W^{\lambda}\right)^{*} \rightarrow K Z(\lambda, m)$. In particular, the images $\psi_{T}$ of the basis vectors $\left[e_{T}\right], T \in \mathcal{T}(\lambda)$, of $\left(W^{\lambda}\right)^{*}=\left(V^{\otimes N} / \mathfrak{n}_{-} V^{\otimes N}\right)_{\lambda}$, form a basis of the space of solutions of the $\mathrm{KZ}$ equation.

Proof. By Lemma 3.6 and Lemma 3.7, $\Psi_{m}^{\lambda}$ is a nonzero homomorphism from the $S_{N^{-}}$ module $\left(V^{\otimes N}\right)_{\lambda}$ to the $S_{N}$-module $K Z(\lambda, m)$. By Theorem $3.1 K Z(\lambda, m)$ is isomorphic to the irreducible $S_{N}$-module $W^{\lambda}$. Since the image of a homomorphism is a submodule, it follows that the map is surjective. On the other hand, by $(2.1), W^{\lambda} \simeq\left(W^{\lambda}\right)^{*}$ occurs in $\left(V^{\otimes N}\right)_{\lambda}$ with multiplicity $\operatorname{dim} M_{\lambda}^{\lambda}=1$ and the claim follows from Proposition 2.2.

Another interesting corollary of Lemma 3.7 is the following classical formula. 
Proposition 3.9. (Frobenius [5]) Let $f_{2}(\lambda)$ be the value of the central element $\sum_{i<j} s_{i j}$ in the representation $W^{\lambda}$. Then

$$
f_{2}(\lambda)=\sum_{b \in \lambda}(c(b)-r(b))
$$

where as before $r(b)$ and $c(b))$ are respectively the row and column coordinates of the box $b$ in the Young diagram $\lambda$.

To prove this we recall that the degree of the polynomial solutions from $K Z(W, m)$ is equal to the value of the central element $\sum_{i<j} m\left(s_{i j}+1\right)$ in the irreducible representation $W$ (see [3]). Comparing this with the leading term of the solution from Lemma 3.7 and taking into account that $\sum_{i<j} 1=\sum_{b \in \lambda}(T(b)-1)=\frac{N(N-1)}{2}$ we come to Frobenius formula (3.2).

\subsection{Integrality}

It is well known (and clear from Proposition 2.1) that $\left(V_{T}\right)_{T \in \mathcal{T}(\lambda)}$ is an integral basis of $W^{\lambda}$, i.e., $W_{\mathbb{Z}}^{\lambda}=\oplus_{T \in \mathcal{T}(\lambda)} \mathbb{Z} V_{T}$ is a module over the group ring $\mathbb{Z} S_{N}$.

Theorem 3.10. The functions $\psi_{T, T^{\prime}}$ are homogeneous polynomials in $z_{1}, \ldots, z_{N}$ with integer coefficients. Thus

$$
\Psi_{m}^{\lambda}\left(z_{1}, \ldots, z_{N}\right)=\sum_{T, T^{\prime} \in \mathcal{T}(\lambda)} \psi_{T, T^{\prime}}\left(z_{1}, \ldots, z_{N}\right) v_{T} \otimes v_{T^{\prime}} \in \mathbb{W}_{\mathbb{Z}}^{\lambda} \otimes_{\mathbb{Z}} W_{\mathbb{Z}}^{\lambda}\left[z_{1}, \ldots, z_{N}\right]
$$

Moreover $\Psi_{m}^{\lambda}$ is $S_{N}$-invariant: $\Psi_{m}^{\lambda}(g \cdot z)=g \otimes g \Psi_{m}^{\lambda}(z)$, for all $g \in S_{N}$.

The invariance is a rephrasing of the homomorphism property of $\Psi_{m}^{\lambda}$ of Theorem 3.8. The integrality follows from repeated application of the following elementary

Lemma 3.11. Let $m_{i}$ be any integers and $w_{1}, \ldots, w_{k}$ distinct complex numbers. Then, for any contour $\gamma$ in the complex plane not passing through $w_{1}, \ldots, w_{k}$,

$$
\frac{1}{2 \pi i} \oint_{\gamma} \prod_{i=1}^{k}\left(t-w_{i}\right)^{m_{i}} d t=\sum_{i<j} c_{i j}\left(w_{i}-w_{j}\right)^{\ell_{i j}}
$$

for some integers $c_{i j}, \ell_{i j}$. 
Theorems 3.8 and 3.10 imply the statements of Theorem 1.1.

Example 3.12. We demonstrate here our formulae in the simplest nontrivial example when $N=3$ and $\lambda=(2,1)$, which corresponds to the usual two-dimensional representation of $S_{3}$. In this case there are two standard tableaux:

$$
T=\begin{array}{l|l}
\hline 1 & 2 \\
\hline 3 & ,
\end{array} \quad S=\begin{array}{l|l|}
\hline 1 & 3 \\
\hline 2 &
\end{array} .
$$

The corresponding primitive vectors are $v_{T}=\epsilon_{3}-\epsilon_{1}, V_{S}=\epsilon_{2}-\epsilon_{1}$.

The residues can be computed explicitly and one obtains a basis of $K Z(\lambda, m)$ :

$$
\begin{aligned}
& \psi_{1}\left(z_{1}, z_{2}, z_{3}\right)=z_{23}^{2 m} \sum_{k=0}^{m} d_{m, k}\left((m-k) v_{T}+k v_{S}\right) z_{12}^{m-k} z_{13}^{k}, \\
& \psi_{2}\left(z_{1}, z_{2}, z_{3}\right)=z_{13}^{2 m} \sum_{k=0}^{m}(-1)^{m-k} d_{m, k}\left((m-k) v_{T}-m v_{S}\right) z_{12}^{m-k} z_{23}^{k},
\end{aligned}
$$

where we abbreviate $z_{i}-z_{j}=z_{i j}$ and

$$
d_{m, k}=-\frac{1}{m}\left(\begin{array}{c}
-m \\
k
\end{array}\right)\left(\begin{array}{c}
-m \\
m-k
\end{array}\right) .
$$

\section{Duality $m \leftrightarrow-m$ and intersection pairing}

To apply our results to the case of negative integers $m$ we can use the isomorphism between the spaces of solutions $K Z(V, m)$ and $K Z(V \otimes$ Alt,$-m)$ mentioned in the Introduction.

Lemma 4.1. For any $\psi \in K Z(V, m)$ the function

$$
\phi=\prod_{i<j}\left(z_{i}-z_{j}\right)^{-2 m} \psi \otimes \epsilon
$$

belongs to the space of solutions $K Z(V \otimes$ Alt, $-m)$.

Indeed from the KZ equation (1.1) we have

$$
\begin{aligned}
\partial_{i} \phi & =(-2 m)\left(\sum_{j \neq i}^{N} \frac{1}{z_{i}-z_{j}}\right) \prod_{i<j}\left(z_{i}-z_{j}\right)^{-2 m} \psi \otimes \epsilon+m \prod_{i<j}\left(z_{i}-z_{j}\right)^{-2 m}\left(\sum_{j \neq i}^{N} \frac{s_{i j}+1}{z_{i}-z_{j}} \psi\right) \otimes \epsilon \\
& =(-m) \prod_{i<j}\left(z_{i}-z_{j}\right)^{-2 m} \sum_{j \neq i}^{N} \frac{s_{i j}+1}{z_{i}-z_{j}}(\psi \otimes \epsilon)=(-m) \sum_{j \neq i}^{N} \frac{s_{i j}+1}{z_{i}-z_{j}} \phi,
\end{aligned}
$$

since $s_{i j}(\phi \otimes \epsilon)=-\left(s_{i j} \phi\right) \otimes \epsilon$. 
Remark 4.2. The pre-factor $\prod_{i<j}\left(z_{i}-z_{j}\right)^{-2 m}$ will disappear if we consider the KZ equation in the form

$$
\partial_{i} \psi=m \sum_{j \neq i}^{N} \frac{s_{i j}}{z_{i}-z_{j}} \psi, \quad i=1, \ldots, N
$$

which in general has rational solutions. The duality between $m$ and $-m$ for similar systems was used in [4] to explain the shift operator for the Calogero-Moser systems.

It is well known that the involution $V \rightarrow V \otimes$ Alt corresponds to the transposition of the Young diagram $\lambda \rightarrow \lambda^{\prime}$. Thus we have established an isomorphism

$$
K Z(\lambda, m) \approx K Z\left(\lambda^{\prime},-m\right)
$$

Note that the configuration spaces $C_{\lambda}\left(z_{1}, \ldots, z_{N}\right)$ and $C_{\lambda^{\prime}}\left(z_{1}, \ldots, z_{N}\right)$ in general are quite different (in particular, they have different dimensions), so the structure of the integral formulas for the solution of the KZ equations for a given Young diagram, which we get in this way, substantially depends on the sign of $m$.

It turns out that there is a link between the spaces of $\mathrm{KZ}$ solutions with the same Young diagram:

$$
j: K Z(\lambda, m) \approx K Z(\lambda,-m)^{*}
$$

More precisely, there exists a natural pairing

$$
K Z(V, m) \times K Z\left(V^{*},-m\right) \rightarrow \mathbb{C}
$$

where $V^{*}$ is the dual space to $V$ with the natural action of $S_{N}$. It is defined by the following lemma.

Lemma 4.3. Let $\langle$,$\rangle denote the canonical pairing between V$ and $V^{*}$. Then for any two solutions $\psi\left(z_{1}, \ldots, z_{N}\right) \in K Z(V, m)$ and $\phi\left(z_{1}, \ldots, z_{N}\right) \in K Z\left(V^{*},-m\right)$ the product $\left\langle\psi\left(z_{1}, \ldots, z_{N}\right), \phi\left(z_{1}, \ldots, z_{N}\right)\right\rangle$ is independent of $z_{1}, \ldots, z_{N}$ and thus defines a nondegenerate canonical pairing (4.3).

The proof is a straightforward check using the KZ equations (1.1):

$$
\partial_{i}\left\langle\psi\left(z_{1}, \ldots, z_{N}\right), \phi\left(z_{1}, \ldots, z_{N}\right)\right\rangle=\left\langle m \sum_{j \neq i}^{N} \frac{s_{i j}+1}{z_{i}-z_{j}} \psi, \phi\right\rangle+\left\langle\psi,(-m) \sum_{j \neq i}^{N} \frac{s_{i j}+1}{z_{i}-z_{j}} \phi\right\rangle=0,
$$


since $\left\langle s_{i j} V, w\right\rangle=\left\langle v, s_{i j} w\right\rangle$ for any $v \in V, w \in V^{*}$. The same calculation holds of course for any Coxeter group.

Note that the $S_{N}$-module $V^{*}$ is isomorphic to $V$ and in the irreducible case the isomorphism is almost canonical in the sense that it is unique up to a scaling factor. This leads to an isomorphism (4.2) and allows us to find the solutions from $K Z(\lambda,-m)$ as follows.

Let us choose any basis $e_{1}, \ldots, e_{M}$ in $V=W^{\lambda}$ and a basis of solutions $\psi_{\alpha}=$ $\sum_{i=1}^{M} \psi_{\alpha}^{i}\left(z_{1}, \ldots, z_{N}\right) e_{i}$, in $K Z(\lambda, m)$. Let $\Phi_{\lambda, m}\left(z_{1}, \ldots, z_{N}\right)=\left\|\psi_{\alpha}^{i}\left(z_{1}, \ldots, z_{N}\right)\right\|$ be the corresponding $M \times M$ fundamental matrix of $K Z(\lambda, m)$. Let $e^{1}, \ldots, e^{M}$ be the dual basis in $V^{*}:\left\langle e^{i}, e_{j}\right\rangle=\delta_{j}^{i}$. We are looking now for a fundamental matrix $\Phi_{\lambda,-m}\left(z_{1}, \ldots, z_{N}\right)=$ $\left\|\phi_{\beta}^{j}\left(z_{1}, \ldots, z_{N}\right)\right\|$ for $K Z(\lambda,-m)$, given by a basis of solutions $\phi^{\beta}=\sum_{j=1}^{M} \phi_{j}^{\beta}\left(z_{1}, \ldots, z_{N}\right) e^{j}$. From Lemma 4.3 it follows that one can choose the basis of solutions in such a way that

$$
\sum_{i=1}^{M} \psi_{\alpha}^{i}\left(z_{1}, \ldots, z_{N}\right) \phi_{i}^{\beta}\left(z_{1}, \ldots, z_{N}\right)=\delta_{\alpha}^{\beta}
$$

Proposition 4.4. A fundamental matrix for $K Z(\lambda,-m)$ can be found as the transposed inverse matrix to the fundamental matrix of $K Z(\lambda, m)$ :

$$
\Phi_{\lambda,-m}\left(z_{1}, \ldots, z_{N}\right)=\left(\Phi_{\lambda, m}\left(z_{1}, \ldots, z_{N}\right)^{-1}\right)^{T} .
$$

The determinant of the fundamental matrix is given by

$$
\operatorname{det} \Phi_{\lambda, m}\left(z_{1}, \ldots, z_{N}\right)=C \prod_{i<j}\left(z_{i}-z_{j}\right)^{2 m d_{+}(\lambda)},
$$

where $C=C(\lambda, m)$ is a nonzero constant and $d_{+}(\lambda)=\operatorname{dim} W_{+}^{\lambda}$ is the dimension of the fixed subspace of reflection $s_{i j}$ acting in the representation $W^{\lambda}$.

The first part is equivalent to (4.4). To prove the formula for the determinant we can use the standard fact that if matrix $\Phi$ satisfies the differential equation $\Phi^{\prime}=A \Phi$ then its determinant satisfies the equation $\operatorname{det} \Phi^{\prime}=\operatorname{tr} A \operatorname{det} \Phi$. Applying this to the KZ equation (1.1) and using the fact that $\operatorname{tr}\left(s_{i j}+1\right)=2 d_{+}(\lambda)$ we have the result. The formula (4.5) shows that the singularities of $\Phi_{\lambda,-m}\left(z_{1}, \ldots, z_{N}\right)$ are located on the hyperplanes $z_{i}=z_{j}$, which of course follows also from the previous considerations.

In the rest of this section we discuss the topological interpretation of the duality (4.3) as intersection pairing between certain homology groups. It is based on the 
integral formula for the solutions of the $\mathrm{KZ}$ equation found in our previous work [3] (see section 4.5).

We restrict ourselves with the special case of the reflection representation of $S_{N}$, which is the standard $(N-1)$-dimensional irreducible representation on the hyperplane $x_{1}+\cdots+x_{n}=0$ in $\mathbb{C}^{N}$ defined by permutation of coordinates. This representation is isomorphic to $W^{\lambda}$ with $\lambda=(N-1,1)$. For positive $m$ our integrals giving the solutions are one-dimensional and, in terms of the standard basis $\epsilon_{b}$ of $\mathbb{C}^{N}$, they have the form

$$
\psi_{a}=\prod_{1 \leq i<j \leq N}\left(z_{i}-z_{j}\right)^{2 m} \operatorname{res}_{t=z_{a}} \prod_{i=1}^{N}\left(t-z_{i}\right)^{-m} \sum_{b=1}^{N} \frac{1}{t-z_{b}} \epsilon_{b} d t, \quad a=1, \ldots, N
$$

They obey the relation $\psi_{1}+\cdots+\psi_{N}=0$ and $\psi_{1}, \ldots, \psi_{N-1}$ form a basis of the solution space $K Z(\lambda, m)$.

A different integral representation for the solution space $K Z(\lambda,-m)$ for positive $m$ was found in [3], where it was shown that

$$
\phi_{a}=\prod_{1 \leq i<j \leq N}\left(z_{i}-z_{j}\right)^{-2 m} \int_{z_{a}}^{z_{N}} \prod_{i=1}^{N}\left(t-z_{i}\right)^{m} \sum_{b=1}^{N} \frac{1}{t-z_{b}} \epsilon_{b} d t, \quad a=1, \ldots, N-1
$$

give a basis in $K Z(\lambda,-m)$. In particular, in the case $N=3$ we have after explicit evaluation of the integrals the following basis:

$$
\begin{aligned}
& \phi_{1}\left(z_{1}, z_{2}, z_{3}\right)=z_{23}^{-2 m} \sum_{k=0}^{m}(-1)^{m+k} d_{m, k}^{\prime}\left((-m-k) v_{T}+k v_{S}\right) z_{12}^{-m-k} z_{13}^{k}, \\
& \phi_{2}\left(z_{1}, z_{2}, z_{3}\right)=z_{13}^{-2 m} \sum_{k=0}^{m} d_{m, k}^{\prime}\left((-m-k) v_{T}+m v_{S}\right) z_{12}^{-m-k} z_{23}^{k},
\end{aligned}
$$

where

$$
d_{m, k}^{\prime}=\left(\begin{array}{c}
m \\
k
\end{array}\right) \frac{(m-1) !(m+k-1) !}{(2 m+k) !}
$$

Thus, in a more invariant geometric terms, for $\lambda=(N-1,1)$ and $m \in \mathbb{Z}_{>0}$, we have two maps

$$
\psi: H_{1}\left(\mathbb{C} \backslash\left\{z_{1}, \ldots, z_{N}\right\}\right) \rightarrow W^{\lambda}, \quad \phi: H_{1}\left(\mathbb{C},\left\{z_{1}, \ldots, z_{N}\right\}\right) \rightarrow W^{\lambda}
$$

sending horizontal sections for the Gauss-Manin connection to solutions in $K Z(\lambda, m)$ and in $K Z(\lambda,-m)$, respectively. The map $\phi$ induces an isomorphism between the complexification of the space of horizontal relative cycles and $K Z(\lambda,-m)$, whereas $\psi$ has a 
one-dimensional kernel spanned by a cycle surrounding all the points $z_{i}$. This kernel is exactly the complexification of the left kernel of the intersection pairing

$$
H_{1}\left(\mathbb{C} \backslash\left\{z_{1}, \ldots, z_{N}\right\}\right) \times H_{1}\left(\mathbb{C},\left\{z_{1}, \ldots, z_{N}\right\}\right) \rightarrow \mathbb{Z}
$$

and the right kernel is trivial. Since the intersection pairing of cycles is preserved by the Gauss-Manin connection we obtain a nondegenerate $S_{N}$-invariant pairing $K Z(\lambda, m) \times$ $K Z(\lambda,-m) \rightarrow \mathbb{C}$.

The claim is that this pairing is proportional to the one described in Lemma 4.3.

Proposition 4.5. Let $\lambda=(N-1,1)$ and $m>0$. The pairing (4.3) of solution spaces $K Z(\lambda, m)$ and $K Z(\lambda,-m)$ is proportional to the image of the intersection pairing $(\cdot)$. More precisely, let $\sigma \in H_{1}\left(\mathbb{C} \backslash\left\{z_{1}, \ldots, z_{N}\right\}\right), \quad \tau \in H_{1}\left(\mathbb{C},\left\{z_{1}, \ldots, z_{N}\right\}\right)$ vary with the points $z_{i}$ as horizontal sections, then

$$
\begin{aligned}
& \left\langle\psi_{\sigma}\left(z_{1}, \ldots, z_{N}\right), \phi_{\tau}\left(z_{1}, \ldots, z_{N}\right)\right\rangle=C_{N} \frac{1}{m}(\sigma \cdot \tau), \\
& \sigma \in H_{1}\left(\mathbb{C} \backslash\left\{z_{1}, \ldots, z_{N}\right\}\right), \quad \tau \in H_{1}\left(\mathbb{C},\left\{z_{1}, \ldots, z_{N}\right\}\right),
\end{aligned}
$$

for some constant $C_{N} \neq 0$ depending on the normalization of the isomorphism $\left(W^{\lambda}\right)^{*} \rightarrow$ $W^{\lambda}$.

Proof. The proof follows from Schur's lemma, except for the determination of the constant of proportionality of the two pairings. To compute it, we consider two special cycles, namely a small circle around $z_{1}$ and a path from $z_{1}$ to $z_{N}$. These cycles have intersection number -1 and the corresponding solutions are $\psi_{1}$ and $\phi_{1}$, respectively. It is sufficient to compute the pairing when $z_{1}=0$ in the limit $z_{N} \rightarrow 0$, where also $\phi_{1}$ is regular. After the change of variable $t=z_{N} \tau$ we obtain

$$
\begin{aligned}
& \psi_{1}\left(0, z_{2}, \ldots, z_{N-1}, 0\right)=F_{m} \operatorname{res}_{\tau=0} \tau^{-m}(\tau-1)^{-m}\left(\frac{\epsilon_{1}}{\tau}+\frac{\epsilon_{N}}{\tau-1}\right) d \tau, \\
& \phi_{1}\left(0, z_{2}, \ldots, z_{N-1}, 0\right)=F_{m}^{-1} \int_{0}^{1} \tau^{m}(\tau-1)^{m}\left(\frac{\epsilon_{1}}{\tau}+\frac{\epsilon_{N}}{\tau-1}\right) d \tau,
\end{aligned}
$$

for some function $F_{m}$ of $z_{2}, \ldots, z_{N-1}$. By integrating by parts we see that the coefficient of $\epsilon_{N}$ is minus the coefficient of $\epsilon_{1}$ (as it should be since the solutions take values in primitive vectors). The result of the calculation is

$$
\begin{aligned}
& \psi_{1}\left(0, z_{2}, \ldots, z_{N-1}, 0\right)=F_{m}(-1)^{m} \frac{(2 m-1) !}{m !(m-1) !}\left(\epsilon_{1}-\epsilon_{N}\right), \\
& \phi_{1}\left(0, z_{2}, \ldots, z_{N-1}, 0\right)=F_{m}^{-1}(-1)^{m} \frac{m !(m-1) !}{(2 m) !}\left(\epsilon_{1}-\epsilon_{N}\right) .
\end{aligned}
$$


If we normalize the pairing $W^{\lambda} \times W^{\lambda} \rightarrow \mathbb{C}$ defining the isomorphism between $W^{\lambda}$ and $\left(W^{\lambda}\right)^{*}$ so that the basis $\epsilon_{a}$ is orthonormal, we obtain $\left\langle\psi_{1}, \phi_{1}\right\rangle=1 / \mathrm{m}$.

Note that since the cycles defining the bases $\psi_{a}$ and $\phi_{a}$ are dual (up to sign) with respect to the intersection pairing, we deduce

$$
\left\langle\psi_{a}\left(z_{1}, \ldots, z_{N}\right), \phi_{b}\left(z_{1}, \ldots, z_{N}\right)\right\rangle=-C_{N} \frac{1}{m} \delta_{a, b},
$$

where $C_{N}=-1$ with the choice of normalization described in the proof.

Remark 4.6. We would like to mention that an extension of these results to the case of general representations should involve a suitable replacement of relative homology. The singularity as integration variables approach each other causes difficulties with the naive generalization.

\section{Acknowledgments}

We are grateful to A. Okounkov, who helped us to trace back the Frobenius formula, to O. Chalykh, who attracted our attention to Opdam's lecture notes [11], and to A. Chervov for stimulating discussions.

This work has been partially supported by the European Union through the FP6 Marie Curie RTN ENIGMA (Contract number MRTN-CT-2004-5652). It was made possible by a visit of A.V. to FIM at ETH, Zurich with the support of the MISGAM programme of the European Science Foundation, and a visit of the authors to the Newton Institute for Mathematical Sciences, Cambridge. We thank these institutions for their support and hospitality.

The work of A.V. was also partially supported by EPSRC (grant EP/E004008/1).

\section{References}

[1] Christe, P., and R. Flume. "The Four-Point Correlations of All Primary Operators of the $d=2$ Conformally Invariant SU(2) $\sigma$-model with Wess-Zumino Term." Nuclear Physics B 282, no. 2 (1987): 466-494.

[2] Feigin, M., and A. P. Veselov. "Quasi-invariants of Coxeter Groups and m-harmonic Polynomials." International Mathematics Research Notices (2002): 521-545.

[3] Felder, G., and A. P. Veselov. "Action of Coxeter Groups On m-harmonic Polynomials and KZ Equations." Moscow Mathematical Journal 3, no. 4 (2003): 1269-1291.

[4] Felder, G., and A. P. Veselov. "Shift Operators for the Quantum Calogero-Sutherland Problems Via Knizhnik-Zamolodchikov Equation." Communications in Mathematical Physics 160 (1994): 259-273.

[5] Frobenius, F. G. Über die Charaktere der symmetrischen Gruppe, 516-534. Berlin: Sitz. König. Preuss. Akad. Wissenschaften zu Berlin, 1900. 
[6] Fulton, W. Young Tableaux, with Applications to Representation Theory and Geometry: Cambridge, UK: Cambridge University Press, 1997.

[7] Goodman, R., and N. R. Wallach. Representations and Invariants of the Classical Groups: Cambridge, UK: Cambridge University Press, 1998.

[8] Kazarnovski-Krol, A. "Cycles for Asymptotic Solutions and the Weyl Group." Pp. 123-150 in The Gelfand Mathematical Seminars, 1993-1995. Boston, MA: Gelfand Math. Sem., 1996.

[9] Macdonald, I. G. Symmetric Functions and Hall Polynomials, 2nd edn. New York, NY: Oxford University Press, 1999.

[10] Matsuo, A. "An Application of Aomoto-Gelfand Hypergeometric Functions to the SU(n) Knizhnik-Zamolodchikov Equation." Communications in Mathematical Physics 134 (1990): 65-77.

[11] Opdam, E. “Complex Reflection Groups and Fake Degrees." In Lectures at RIMS, Kyoto University (Japan) in 1997. (1998): Preprint arXiv: math.RT/9808026.

[12] Varchenko, A. "Hypergeometric Functions and Representation Theory of Lie Algebras and Quantum Groups." In Advanced Series in Mathematical Physics, Vol. 21. River Edge, NJ: World Scientific, 1995.

[13] Zamolodchikov, A. B., and V. A. Fateev. "Operator Algebra and Correlation Functions in the TwoDimensional $S U(2) \times S U(2)$ chiral Wess-Zumino Model." Soviet Journal of Nuclear Physics 43 (1986): 657-664. 